\title{
Technological solution for determining gait parametersusing pressure sensors: A case study of multiple sclerosis patients
}

\author{
María Viqueira Villarejo ", Jose Maeso García, Begoña García Zapirain and Amaia Méndez \\ Zorrilla \\ Deustotech Life Department, University of Deusto, Bilbao 48007, Spain
}

\begin{abstract}
This paper describes a study dealing with a technological solution to measure gait quality in people suffering from multiple sclerosis (MS) by selecting objective parameters that focus on their step. Android mobile technology, online services and four wireless pressure sensors are used in concert for this purpose. The objective of this work is the early detection of deterioration of the patient so that a physician can quickly intervene. Tests were carried out on a group of 8 persons with MS, and these results were compared with a control a group of 6 healthy participants. The results indicated a statistical difference in 7 of 40 general step features, with a minimum $\sigma=0.013$ and a maximum $\sigma=0.029$. These characteristics showed differences between first and fifth metatarsals for each group. It was concluded that these parameters can be used to evaluate gait degeneration in people with MS and that further information could be obtained from measurements with sensors to monitor activities such as bending and inertial sensors.
\end{abstract}

Keywords: Gait analysis, objective parameterization, sensors

\section{Introduction}

A great many studies are currently being conducted to assess gait quality for a variety of reasons. As examples, athletes who wish to analyse and improve their performance, patients undergoing rehabilitation, patient posture correction to avoid back pain, etc. Abnormal walking can be caused by various factors ranging from poor posture or habits [1], foot shape [2], improper footwear, back problems and even neuronal diseases [3]. At present, physicians evaluate patients who suffer from neurodegenerative diseases such as Multiple Sclerosis (hereinafter MS) by using brief performance tests. The brevity of these tests makes it difficult to obtain objective concise information about a patient's condition. In this regard, gait evaluation is highly prominent, because the various stages of neurodegenerative diseases include symptoms that affect a patients' capacity to walk and the number and frequency of pauses made during walking. Gait evaluation can be combined with specific information about the quality of the patient's step over the distance travelled. During the life cycle of the disease, MS patients lose the capacity to walk under controlled conditions. Thus, by measuring select parameters over

${ }^{*}$ Corresponding author: María Viqueira Villarejo, Deustotech Life Department, University of Deusto, Bilbao 48007, Spain. Tel.: +34-944-139000 ext. 2051; Fax: +34-944-139101; E-mail: mviqueira@deusto.es.

0959-2989/14/\$27.50 @ 2014 - IOS Press and the authors. 
time, it is possible to determine the evolution of a patient's condition and thereby how best to modify the medication and rehabilitative treatment initially prescribed.

This paper presents a technological application that integrates a mobile technique, an online platform and pressure sensors to analyze and store data about a patients' step over long time periods. Information about actual MS sufferers was obtained through collaboration with the Biscay Multiple Sclerosis Association (ADEMBI). The same system was applied to healthy individuals, which is referred to as the control group.

A series of characteristics was collected from the signals emitted by the sensors and statistical analysis of the MS group and control group was carried out using the Mann-Whitney system.

The system described consisted of three different parts: a hardware device placed on an insole that stores and sends data; a mobile device which collects the data by using an application and lastly, an online technology platform where the acquired parameters are processed and the results are displayed to assist the specialist during the monitoring of the patient. Simultaneously, the patient can access his/her own information on the mobile device or the online platform.

\section{Background}

At the present time, numerous studies are being conducted on the gait of persons with different diseases, including neuronal diseases. The possibility of using a device to help medical specialists monitor these patients is being explored. Developments in wireless sensors to measure pressure have proven promising. There is no doubt about their potential use, especially in biomechanics and in aiding diagnosis and evaluation.

The study described in reference [4] presents a method to check the fatigue in MS patients by using a camera and infrared-based system which measures parameters such as step length, height and width, flexion angle, etc.

Shu et al. [5] developed a system to measure and analyze foot plantar pressure using a sensor integrated in the shoe. This sensor was a matrix of textile pressure sensors, which renders them soft, light and extremely sensitive. The study by Saito et al. [6] also cited several advantages, including the avoidance of placing a data processor or microcontroller in the shoe, which helped to make their system smaller. Other advantages include lower energy consumption and greater autonomy. However, these improvements have affected accuracy, yielding a maximum of $250 \mathrm{kPa}$, while a typically obese individual reaches pressure spikes of $500 \mathrm{kPa}$.

In [7] the authors describe an insole that integrates 48 pressure sensors and triple axis accelerometer, gyroscope and magnetometer. The sensor signals are sent to a smart phone. Barth et al. [8] used a combination of accelerometers and gyroscopes to analyze and classify the gait in patients with Parkinson's Disease (PD). A wearable sensor system is described in reference in [9] to analyze gait during the Timed up and Go (TUG) test with PD patients. These authors also used a combination of accelerometers and gyroscopes to obtain information.

Continuing with the tests conducted on PD patients, a recent study centered on sensors worn on the waist to analyze posture and gait [10]. The information was sent to a smartphone where it was processed to be used later with the other results to determine the best treatment.

B.R. Greene et al. [11-13] provided a method for objective assessment of fall risk in older adults using quantitative analysis of gait and turning employing body-worn inertial sensors. This system used body-worn tri-axial gyroscopes streaming over Bluetooth to a touch screen mobile device. Statistics 
were displayed on the mobile device, providing a comprehensive assessment of a patient's mobility as well as a comparison against the normal range for age and gender.

Another study [14] was based on the body pressure center (PC), related to foot plantar pressure. The results of this study indicated clear differences when comparing the pressure center of healthy adults with adults suffering from MS.

The study described in reference [15] describes the major dissimilarities between patients suffering from MS and control subjects of the same age and sex. The main disparity between the two groups was that patients diagnosed with MS walked more slowly, taking wider shorter steps while keeping both feet on the floor longer during the gait cycle. One of the main details was that the variability of these characteristics was much higher in MS patients than in the control group. This fact makes quantification of this variability crucial, in tracking MS patients regardless of absolute data. In the study detailed in reference [16] it was concluded that patients with MS have a repetitive, but less adaptable manner of walking. An inflexible gait mode does not help MS patients to adjust their manner of walking to their surroundings and their manual tasks at a given moment.

As stated previously, this experimental system includes wearable sensors that send information via Bluetooth. Similarly, reference [17]describes a study conducted using wearable, wireless sensors.

\section{Methods}

\subsection{Gait signal processing algorithm}

The algorithm used to process the signals from the sensors was based on are ported study report [18], where steps were detected using a mobile accelerometer. This algorithm defines a standard step time of 1.2 seconds, taking the center of the signal \pm 0.6 seconds as the initial search range for the first maximum signal strength. From this maximum, it displaces 1.2 seconds along the signal path. It checks values at 0.1 seconds to the left and 0.1 seconds to the right to locate a new maximum during each displacement. If the located maximum is in the first third of the search window, the maximum local strength is estimated to be established outside the search zone, so that the search zone is enlarged towards the left. If the maximum is located in the last third of the window, the same result occurs, but the search zone is enlarged towards the right. If the maximum is located in the second third of the window (center), it is considered to be the valid maximum signal. The search continues in the next window from the final maximum strength.

\subsection{Arduino and mobile application}

An Arduino platform was used. The model chosen was the Arduino mini 05 with an Atmega328 processor. Four of its analog inputs were used to take the measurements of the pressure sensors with a sampling frequency of $20 \mathrm{~Hz}$. Communication between the device and the mobile receptor was conducted via Bluetooth.

An Android OS was used for the mobile application. The Android framework included various parts from the core of the device which offered integration and easy programming mechanisms such as those from the operating system itself to the middleware and main programs. Besides the programming facilities that Android offers, its use has increased in recent years, because it has been installed in many types of devices, from the simplest to the most complex, reaching all types of users. 


\subsection{Server and others}

The server where the information was stored was divided into two parts. The first part was the database where the acquired sensor information was stored. Due to its simple use and large user community, a MySQL database was used for this purpose. The second part was an applications server where the various applications that are constantly functioning are run such as the web and integration module. Java was chosen as the language for the entire system to achieve a homogeneous programming language, also used in the Android, using JSF (Java Server Faces) and JBoss as application server.

Finally, a Representational State Transfer (REST) type Application Programming Interface (API) was chosen as integration module.

The mathematical calculation program Matlab was used to program the local interface as well as the initial design of the step algorithm. This allowed for acquisition of the table of characteristics that would be analyzed with the statistical calculus program SPSS v20 by using Mann - Whitney.

\section{System design}

This section describes the different blocks that make up the system and the various parts of each block:

1. Sensors used and assembly diagram.

2. The data from the sensors captured by the mobile as well as the information and use of the GPS.

3. Webpage functions and users and user interface

4. Research system.

The main architecture of the system can be seen in Fig. 1. The processor acquires the signals from the sensors and sends them via Bluetooth to a smartphone, which stores the data and sends them to the server. The server processes the data to obtain objective gait parameters, particularly the step parameters. This information is used to draw up reports about patients on a particular day, as well as their case history and evolution.

As can be seen in the Figure $1 \mathrm{~b}$ above, there is a green module which was used to study the acquired data. The purpose of this module was to facilitate development of the main system by comparing the dissimilar characteristic data obtained for the two groups participating in the study.
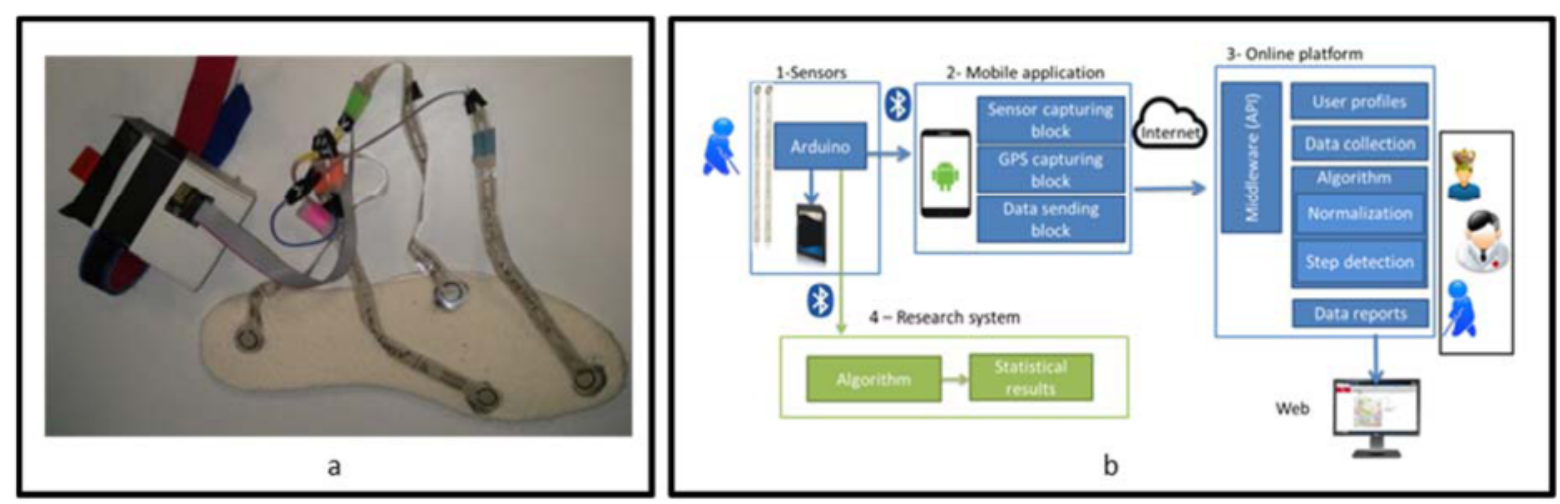

Fig. 1. a. Placement of the different sensors and hardware system. B. General scheme. 
The following sections describe the modules that comprised the system and their parts and functions.

\subsection{Sensors and mobile application}

Four Flexiforce 25lb. load cells were placed in various insoles as shown in Fig. 1. One load cell was placed under the hallux, two more under the first and fifth metatarsal and a forth under the heel. The signals were acquired with a sampling frequency of $20 \mathrm{~Hz}$.

The user interacted with the data capture system using an application on the mobile device. The $\mathrm{v}$ 4.0 Android operating system was employed on the mobile device. The sensor capturing block captured data from the server and was initiated by pressing a button on the first screen of the application. GPS sensors were used rather than sensors integrated in the insole. The data sending block allowed the device to use an Internet connection via either $3 \mathrm{G}$ or $\mathrm{Wi}-\mathrm{Fi}$, to upload the information to the server using the API REST implemented within the system.

\subsection{Online platform}

Once the data were collected, they were displayed on a web application that was accessible to the patient and the doctor. Web application users are divided into doctors, patients and administrators.

\subsubsection{Data collection}

The server used the API (explained in the following section) to capture the information sent by the mobile application. It received a chain of characters with the information sent from the sensors and parsed this so that could be read and processed.

\subsubsection{Algorithm}

This included a normalization phase and another data analysis phase to detect the steps.

Normalization: different values from the analog input of Arduino were obtained and the pressure sensor values were replaced with actual resistance measurements. The resistance reading for a weight of $25 \mathrm{~kg}$ is approximately $50 \mathrm{k} \Omega$. The manufacturer recommends calibrating the device using conductance rather than resistance, because the conductance output is linear with applied force. Output relationship between force and conductance is defined as:

$$
\text { force }=75610^{*} \text { values }^{-1.674}
$$

The final normalization data provides the force each user applies in relation to his/her total weight. Thus, an $89 \mathrm{~kg}$ person who applies force of $8 \mathrm{~kg}$ will be apply $10 \%$ of his/her total weight.

b) Step detection: there are four different vectors for step detection. These correspond to the values generated by the load cells $\{\mathrm{v} 1: \mathrm{v} 4\}$. This is based on a person using approximately 1.2 seconds to take a step. First, the central sample $\left(\mathrm{X}_{\mathrm{c}}\right)$ of a total number of samples $(\mathrm{N})$ of the vector $(\mathrm{v})$ are located. Next, a sample margin equal to 1.2 times half the sample frequency $(\mathrm{M})$ is obtained and $\mathrm{M}$ samples are taken both to the right and left of $X_{c}$, which will be the first interval $\left(\mathrm{F}_{\mathrm{i}}\right)$ where the sample base is located $\left(X_{b}\right) . X_{b}$ will be the maximum value of the interval $F_{i}$.

$$
\mathrm{Xc}=\frac{\mathrm{N}}{2}
$$




$$
\begin{aligned}
& F i=v\{X c-M: X c+M\} \\
& X b=\max \{F i\}
\end{aligned}
$$

The next sample $\left(X_{n}\right)$ was then taken at a distance of $d=2 \cdot M$ from $X_{b}$ on the left and the same previously defined margin is taken once again, locating the maximum value of the interval $\left(\mathrm{S}_{\max }\right)$ for its storage again. The algorithm repeats the process iteration through from $X_{n}$ until it reaches the beginning of $v$. Finally, the same operations are performed to locate the maximum values found to the right of $X_{b}$.

$$
\begin{aligned}
& \operatorname{Smax}_{\text {left }}(i)=\max \{(X b-i \cdot d-M):(X b-i \cdot d+M)\} \forall(X b-i \cdot d-M)>0 \\
& \operatorname{Smax}_{\text {right }}(i)=\max \{(X b+i \cdot d-M):(X b+i \cdot d+M)\} \forall(X b+i \cdot d+M)<N \\
& \operatorname{Smax}_{=}\left[\operatorname{Smax}_{\text {left }}, \operatorname{Smax}_{\text {right }}\right]
\end{aligned}
$$

After performing the previous operations, a check is run to determine if there is a maximum value between two consecutive maximum values. Once the maximum values have been calculated, the same operations are performed to locate the minimum values for each interval.

$$
\begin{aligned}
& \operatorname{Smin}_{\text {left }}(i)=\min \{(X b-i \cdot d-M):(X b-i \cdot d+M)\} \forall(X b-i \cdot d-M)>0 \\
& \operatorname{Smin}_{\text {right }}(i)=\min \{(X b+i \cdot d-M):(X b+i \cdot d+M)\} \forall(X b+i \cdot d+M)<N \\
& \operatorname{Smin}=\left[\operatorname{Smin}_{\text {left }}, \operatorname{Smin}_{\text {right }}\right]
\end{aligned}
$$

Finally, a check is run to see if there are two consecutive maximum or minimum values and the lowest or highest are deleted. Once these have been deleted, two new vectors, $V_{\max }$ and $V_{\min }$ are created. They only contain the maximum and minimum values whose difference is 1.5 times higher than the standard deviation.

\subsubsection{Data reports}

The graphs of the evolution of gait speed, steps, duration of stops, etc. for a sample of the last six months are displayed. Additional graphs can be displayed by pressing on each distance travelled. These include the number of stops, duration of each stop, relationship between time walking and time stopped and the complete progress of the distance travelled shown as a line with different colors indicating the time walking and standing still or the percentage of each one. Physicians can check the report via the web or create a PDF file with the information collected by the application.

\subsubsection{Application programming interface}

An API was designed to connect the mobile device application with the server's database, from which the webpage later obtains the information and displays it to doctors and patients. This interface is based on HTTP type requests with pre-established formats and differentiates the use depending upon the method within the HTTP protocol. 


\subsection{Research system}

In order to check for variations between the two groups in the study, a statistical analysis comparing different walking gait characteristics was performed. This algorithm block included the normalisation stage and a step detection algorithm. The difference between these is that it was developed using Matlab to facilitate data analysis. The statistical results block does the following: once the steps have been clearly differentiated, the highest spikes obtained for each are used to find a series of characteristics such as the force exerted, step time, deviation between spikes, etc.

\section{Results}

A group of individuals who were diagnosed with MS (4 women, 4 men) formed the MS group and 1 woman and 5 men without any diagnosed disease comprised the control group. The participants' ages ranged from 21 to 53 . They were requested to use comfortable and flat shoes to ensure that the shoes were similar among all subjects. This minimized the noise that different shapes of shoes could generate.

Once the insole was placed and the sensors attached to their legs, participants were asked to walk an imaginary 7.5 meter straight line, which had been previously measured and marked on the floor. The tests were conducted indoors under ambient conditions. Participants were required to accomplish this task four times without stopping, turning around each time. They were asked to walk normally.

Once the test had been completed, participants were asked to complete a questionnaire on the system. After having obtained the samples, each of the user's steps was studied to obtain the characteristics that were then statistically analyzed using Mann-Whitney.

Fig. 2 shows the different steps detected for each of the four signals coming from the pressure sensors for a patient with MS.

The maximum values denote the moment at which the user placed the sole of his/her foot on the floor (flatfoot stage) and the minimum values depict when the foot is not on the floor.
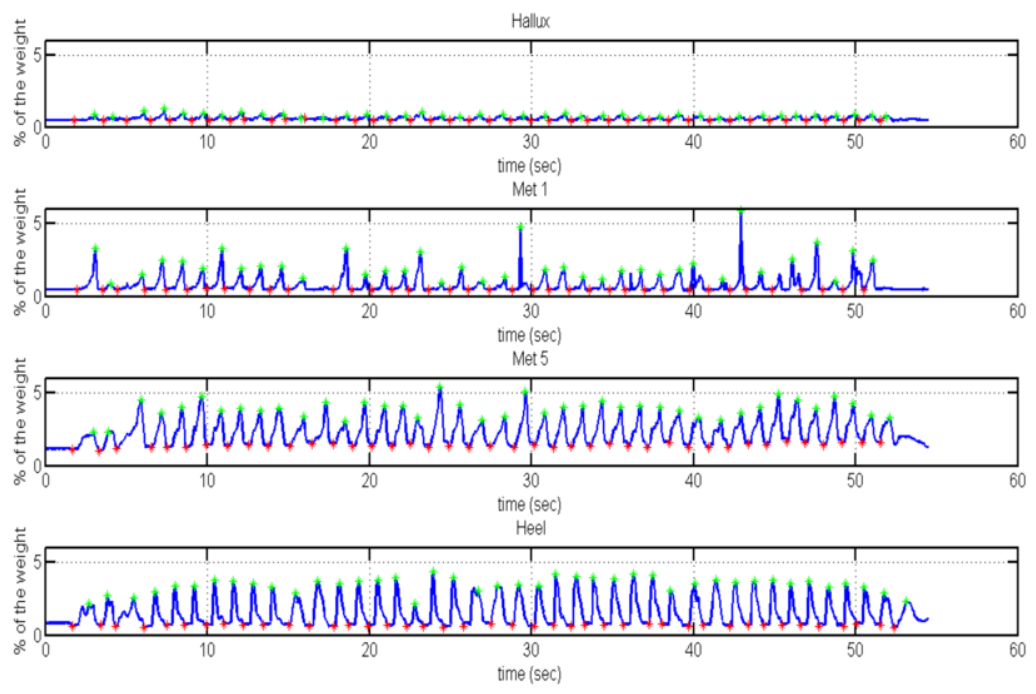

Fig. 2. Step detection in a patient with MS 
Table 1 Sigma results for Mann Whitney

\begin{tabular}{|l|l|l|l|l|}
\hline & Hallux & Metatarsal 1 & Metatarsal 5 & Heel \\
\hline C1 & 0.2820 & 0.2280 & 0.0290 & 0.0590 \\
\hline C2 & 0.5730 & 1.0000 & 0.3450 & 0.2280 \\
\hline C3 & 0.5730 & 0.1810 & 0.0200 & 0.2280 \\
\hline C4 & 0.4910 & 0.1420 & 0.0130 & 0.2280 \\
\hline C5 & 1.0000 & 0.9500 & 0.8520 & 1.0000 \\
\hline C6 & 0.4140 & 0.1080 & 0.0130 & 0.1420 \\
\hline C7 & 0.9500 & 0.0200 & 0.1420 & 0.2820 \\
\hline C8 & 0.4140 & 0.1420 & 0.4910 & 0.4140 \\
\hline C9 & 0.9500 & 0.0200 & 0.0590 & 0.3450 \\
\hline C10 & 0.2280 & 0.0590 & 0.0290 & 0.2820 \\
\hline
\end{tabular}

A study of the information and characteristics of the MS patients and the control group when walking indicated that MS patients press their feet harder and do not control their foot movements very well. This latter information indicated there may be a difference in step variability.

Data were analyzed using Matlab and the specific algorithm, which creating graphs of the steps. After differentiating steps, various strength spikes were used to obtain the different characteristics which were analyzed with the SPSS program using Mann-Whitney.

Ten characteristics were obtained for each of the 20 users and each of the 4 signals. In addition,

40 characteristics were analyzed for each person. These included: The average of: signal (C1), minimum values $(\mathrm{C} 2)$, maximum values $(\mathrm{C} 3)$, amplitude between the maximum and minimum values (C4), step time (C5)

- Standard deviation of the signal (C6) and maximum values (C10),

- Area between: two minimum values (C7), two minimum values * height (C8), two minimum values * width (C9),

The reason for studying the area of each peak of the signal was to determine if the relation between step time and force applied was longer for one group than for the other. The same area value can appear with different widths and heights. Therefore, a multiplication factor was used to place more weight on the step height and width.

The deviation between the maximum values was studied, because patients with MS show a higher variability when walking [15]. There is usually a high deviation between the different forces applied when stepping. The previous characteristics were analyzed in a nonparametric study (Mann - Whitney). There appeared to be a difference between the two groups if $\sigma<0.05$ was obtained.

Table 1 shows that there is a significant difference in the first metatarsal $(\sigma$ Met $1 \mathrm{C} 7=0.0200$, $\sigma$ Met1C $9=0.0200)$ and in the fifth metatarsal $(\sigma \mathrm{Met} 5 \mathrm{C} 1=0.0290, \sigma \mathrm{Met} 5 \mathrm{C} 3=0.0200, \sigma \mathrm{Met} 5 \mathrm{C} 4=$ $0.0130, \sigma \mathrm{Met} 5 \mathrm{C} 6=0.0130, \sigma \mathrm{Met} 5 \mathrm{C} 10=0.0290)$. The third characteristic, the average of maximum values, refers to the mean of the moments when most force is applied. The fourth characteristic is the amplitude between the maximum and minimum values, referring to the force difference in the same step from the moment the foot is placed on the floor until it is back in the air.

According to Fig. 3, characteristic $\mathrm{C} 5$, the time between each step, gives very high $\sigma$ values $(\sigma$ HallucC $5=1.0000, \sigma$ Met $1 \mathrm{C} 5=0.9500, \sigma$ HeelC $5=1.0000)$, which demonstrates that the conducted tests show no significant difference.

Fig. 3 shows a bar graph comparing the key characteristics. The areas obtained in the first metatarsal are shown as are the characteristics obtained for the fifth metatarsal. 

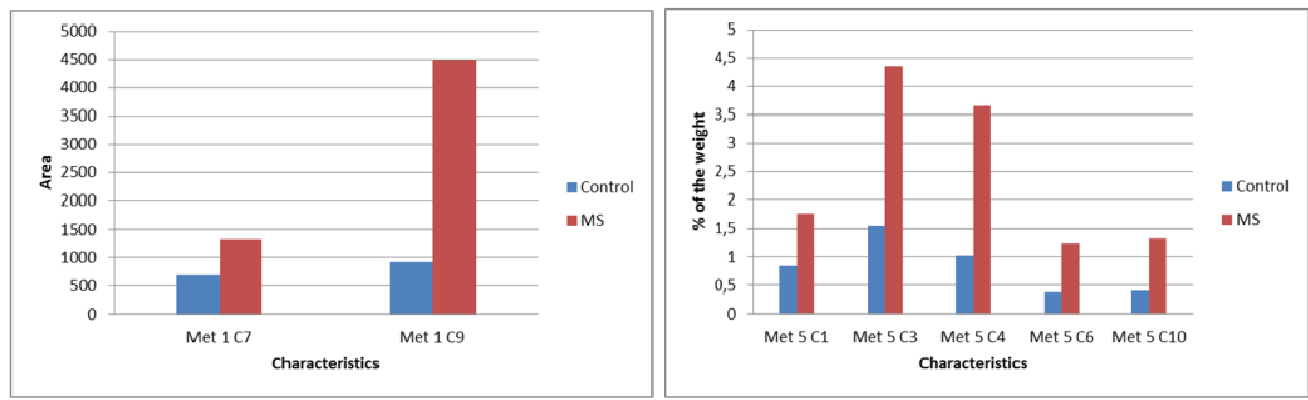

Fig. 3. Comparison between averages for the different characteristics and the two groups.

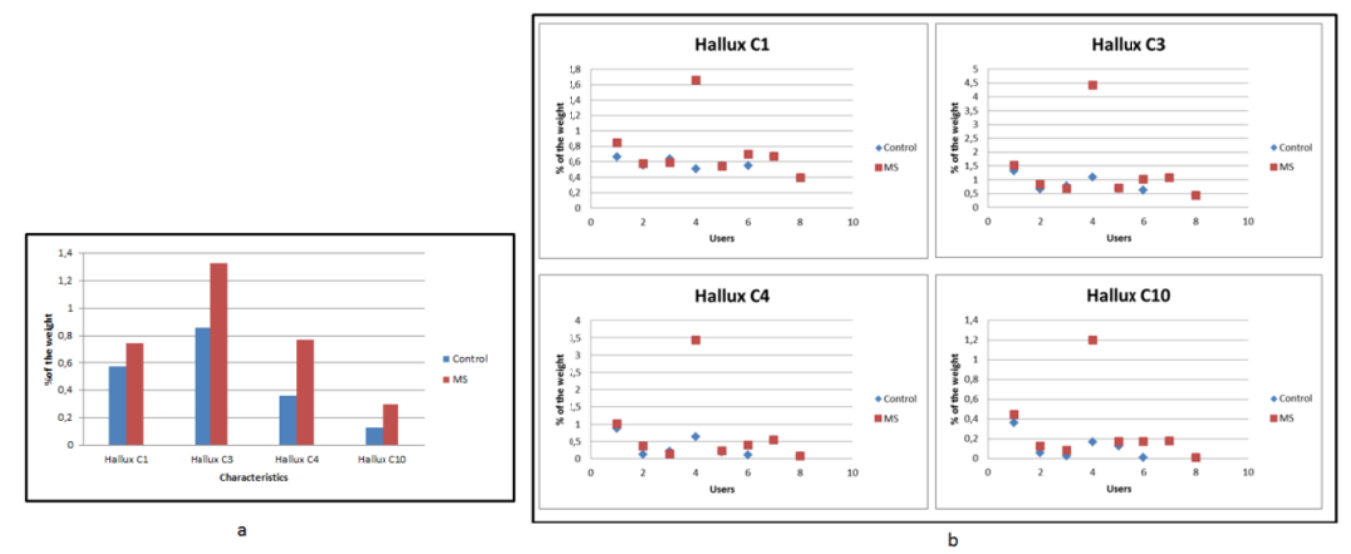

Fig. 4. a) Characteristics of the hallux and b) Values of hallux characteristics.

As shown in the graph, characteristic 7 of the first metatarsal gives a value $91 \%$ higher than the control group characteristic 9shows increase of $492 \%$. It is the fifth metatarsal that exhibits most significant differences, with higher levels being recorded for the MS group. The first metatarsal shows variations in the areas for each step.

The members of the control group showed similar values in each characteristic (deviations: 0.1455 , $0.3476,0.1675,0.2736$ ) while there were greater differences among members of the MS group (deviations: $0.8276,2.5917,0.8472,1.0598$ ) and they obtained higher values than the control group. There is no significant difference between the two groups for the hallux, although the Fig. 4 shows higher values for MS patients.

A breakdown of the values for the subjects' characteristics, Fig. 4 indicates that both groups have a similar value and user 4 in the MS group was the subject most responsible for the average value to rise.

\section{Discussion and conclusion}

This study presents a technological solution to analyse and store data on patients' step which integrates a mobile application, an online platform and pressure sensors to analyse and store data over long periods of time. Since this system can be inserted in any type of shoe, it enables doctors to obtain an objective assessment of a patient's walking routine in different situations throughout the day. 
An experiment was conducted using the subject device to evaluate MS patients' walking along a using the 7.5 meter test distance that could be applied in a doctors' office. A quantitative study of the patients' step was performed to assist the doctor with objective evaluation information. Conducting short tests made it possible for patients with different stages of the disease to participate. This study revealed information about the patients' step whereas other [19] provide information about how MS patients walking along in the 7.5 meter test, comparing them with a control group.

The following section provides an in-depth review of each of the key points from the analysis of the results. The differences in force applied on the fifth metatarsal were analysed, with the statistical results and future areas of research.

It was observed that five of the MS patients applied a greater force on the fifth metatarsal when stepping (between $1.3902 \%$ and $8.7861 \%$ of the force applied with each step) than the control group $(1.1364 \%-2.1484 \%)$.

After running Mann-Whitney on these data, it was observed that substantial differences were found for the first and fifth metatarsals, which demonstrated a greater difference at the moment when the foot is placed on the floor. The following section explains the various parameters obtained in Table 1 .

Significant differences were only found for the first and fifth metatarsals while none was found for the hallux and heel. Therefore, the main differences occur at the moment the subject's foot is placed on the floor.

For the first metatarsal, the substantial differences were found for $\mathrm{C} 7$ (the area beneath each step) and C9 (the area beneath each step multiplied by height). Both have a sigma value of 0.0020 , which indicates a significant difference between the two groups. These characteristics demonstrate a relationship between the force applied when stepping and the time used for each step, which is greater in the case of MS patients. Multiplying the area obtained for height as well as for width allows one to determine if the area difference between the two groups is due to the force they apply when stepping or the time used for each step. In this case, the difference was observed in C9 and not in C8 (area multiplied by width), showing that main difference is due to the force applied and not the time used for each step.

The fifth metatarsal showed differences in the peak average and force exerted when stepping $(\sigma \mathrm{Met} 5 \mathrm{C} 1=0.0290, \sigma \mathrm{Met} 5 \mathrm{C} 3=0.0200)$, in the amplitude of maximum and minimum values, $(\sigma \mathrm{Met} 5 \mathrm{C} 4=0.0130)$ and in the variability of the signals $(\sigma \mathrm{Met} 5 \mathrm{C} 6=0.0130)$ as well as the different pressure points $(\sigma \mathrm{Met} 5 \mathrm{C} 10=0.0290)$. The force applied $(\mathrm{C} 1, \mathrm{C} 3$ and $\mathrm{C} 4)$ was found to be greater in MS patients. The results for variability coincide with previous reports[15], where the step variability for persons with MS was found to be higher than in the control group.

Very high $\sigma$ values are shown for the time between each step, $(\sigma$ HalluxC5 $=1.0000, \sigma$ Met1C $5=$ $0.9500, \sigma \mathrm{HeelC} 5=1.0000$ ), which demonstrates that there is no significant difference in the tests run. It is assumed that this result occurred because the test was too short and did not adequately demonstrate the MS patients' fatigue, which would occur when walking for longer periods of time. Some differences would probably result if tests were conducted for longer periods of time.

The present study uses the Mann - Whitney statistical test because of the small sample size (as was the case with a previous study [19]), whereas in a recent report [15] the independent samples t-test was used to compare select parameters and detect differences between the MS patients and the control group. In this reported study, the GAITRiteTM analysis system was used. However, it is limited to use for short distances indoors while the system proposed in this paper can be applied to long distances outdoors by using wireless technologies.

Other studies combine pressure sensors and mobile applications [21], which only shows the value of the pressure applied, but calculates with the data, showing parameters such as the center of pressure 
(COP). In the present study, parameters were displayed on the web and the mobile device which was not the case in other studies. Examples include duration of stops, number of stops, evolution, etc.

The following key points can be drawn as the conclusions of this study:

- The two areas of the foot studied were the first and fifth metatarsals, for MS patients and the control group.

- For the first metatarsal, the characteristics studied to determine the test subjects' evolution were the area between two minimum values and the area between two minimum values multiplied by the width.

- The following characteristics should be studied for the fifth metatarsal: the average signal, the average of the maximum values, the average amplitude between maximum and minimum values, standard deviation of the signal and the maximum value deviation. The characteristics $\mathrm{C} 4$, the average amplitude between the maximum and minimum values, and C6, standard deviation of the signal are especially interesting because they show lower $\sigma$ values.

- The following characteristics did not prove to be relevant for study of the human gait in any of the cases: $\mathrm{C} 2$, average minimum value, $\mathrm{C} 5$, average step time and $\mathrm{C} 8$, area between two minimum values multiplied by height.

- The time is not significant because the tests were run over very short time periods and the differences are very small.

Lastly, the following topics of research should be considered in the future to further develop this area of research:

- Replicate the test over longer distances. This would eliminate those MS patients in advanced stages of the disease who are incapable of walking long distances.

- Repeat the tests with a larger sample spaces, i.e. larger number of patients and other groups such as subjects with Parkinson's disease or the elderly.

- Insert more pressure sensors on the floor to include more pressure points and also include angle sensors to detect the arch of the foot.

- Adapt the system to other platforms such as iOS or Windows Phone.

\section{Acknowledgement}

This Project has been partially founded by the Department of Education of the Basque Country, by the project CTP - 2012/P05. We thank ADEMBI and Alejandro Basterra for support.

\section{References}

[1] P. Thompson, Disorders of posture and gait, Journal of Neurology, Neurosurgery, and Psychiatry, BMJ Group 50 (1987), 507.

[2] D.B. Thordarson, Foot and Ankle, Lippincott Williams \& Wilkins, USA, 2004, pp. 3-33.

[3] J.-J. Z., X.-B. Ning, X.-D. Y., F.-Z. H. and C.-Y. H., Decrease in hurst exponent of human gait with aging and neurodegenerative diseases, Chinese Phys. B 17 (2008), 852-856.

[4] A. Sehle, A. Mündermann, K. Starrost, S. Sailer, I. Becher, C. Dettmers and M. Vieten, Objective assessment of motor fatigue in multiple sclerosis using kinematic gait analysis: A pilot study, J. Neuroeng. Rehabil. 8 (2011), 1530-1539.

[5] A.H.A. Razak, A. Zayegh, R.K. Begg and Y. Wahab, Foot plantar pressure measurement system: A review, Sensors (Basel) 12 (2012), 9884-9912. 
[6] M. Saito, K. Nakajima, C. Takano, Y. Ohta, C. Sugimoto, R. Ezoe, K. Sasaki, H. Hosaka, T. Ifukube, S. Ino and K. Yamashita, An in-shoe device to measure plantar pressure during daily human activity, Med. Eng. Phys. 33 (2011), 638-645.

[7] W. Xu and J.J. Liu, Smart insole: A wearable system for gait analysis, Proceedings of the 5th International Conference on Pervasive Technologies Related to Assistive Environments, 2012, 1-4.

[8] J. Barth, M. Sünkel, K. Bergner, G. Schickhuber, J. Winkler, J. Klucken and B. Eskofier, Combined analysis of sensor data from hand and gait motor function improves automatic recognition of Parkinson's disease, Conf. Proc. IEEE Eng. Med. Biol. Soc., 2012, 5122-5125. doi: 10.1109/EMBC.2012.6347146

[9] B. Mariani and C. Jim, On-shoe wearable sensors for gait and turning assessment of patients with parkinson's disease 60 (2013), 155-158.

[10] D. Rodriguez-Martin, A. Samà, C. Perez-Lopez, A. Català, J. Cabestany and A. Rodriguez-Molinero, SVM-based posture identification with a single waist-located triaxial accelerometer, Expert Syst. Appl. 40 (2013), 7203-7211.

[11] B.R. Greene, E.P. Doheny, A. O'Halloran and R. Anne Kenny, Frailty status can be accurately assessed using inertial sensors and the TUG test, Age Ageing 0 (2013), 1-6.

[12] B.R. Greene, E.P. Doheny, C. Walsh, C. Cunningham, L. Crosby and R. a Kenny, Evaluation of falls risk in community-dwelling older adults using body-worn sensors, Gerontology 58 (2012), 472-480.

[13] B.R. Greene, A. O’Donovan, R. Romero-Ortuno, L. Cogan, C.N. Scanaill and R. a Kenny, Quantitative falls risk assessment using the timed up and go test, IEEE Trans. Biomed. Eng. 57 (2010), 2918-2926.

[14] J.M. Abrantes and L.F. Santos, Plantar pressure assessment: A new tool for postural instability diagnosis in multiple sclerosis, in: Technologies for Medical Sciences, Springer, Netherlands, 2012, pp. 179-204.

[15] J.J. Sosnoff, B.M. Sandroff and R.W. Motl, Quantifying gait abnormalities in persons with multiple sclerosis with minimal disability, Gait Posture 36 (2012), 154-156.

[16] J.P. Kaipust, J.M. Huisinga, M. Filipi and N. Stergiou, Gait variability measures reveal differences between multiple sclerosis patients and healthy controls, Motor Control 16 (2012), 229-244.

[17] T. Tanantong, E. Nantajeewarawat and S. Thiemjarus, Toward continuous ambulatory monitoring using a wearable and wireless ECG-recording system: A study on the effects of signal quality on arrhythmia detection, Bio-Medical Materials and Engineering 24 (2014), 391-404.

[18] M.O. Derawi, P. Bours and K. Holien, Improved cycle detection for accelerometer based gait authentication, 2010 Sixth International Conference on Intelligent Information Hiding and Multimedia Signal Processing, 2010, 312-317.

[19] R.I. Spain, R.J. St George, a. Salarian, M. Mancini, J.M. Wagner, F.B. Horak and D. Bourdette, Body-worn motion sensors detect balance and gait deficits in people with multiple sclerosis who have normal walking speed, Gait Posture 35 (2012), 573-578.

[20] M. a Gianfrancesco, E.W. Triche, J. a Fawcett, M.P. Labas, T.S. Patterson and A.C. Lo, Speed-and cane-related alterations in gait parameters in individuals with multiple sclerosis, Gait Posture 33 (2011), 140-142.

[21] L. Shu, T. Hua, Y. Wang, Q.L. Q., D.D. Feng and X. Tao, In-shoe plantar pressure measurement and analysis system based on fabric pressure sensing array, IEEE Trans. Inf. Technol. Biomed. 14 (2010), 767-775. 